Mots. Les langages du politique

$111 \mid 2016$

Normes et usages de la langue en politique

\title{
Le volcanique Léon Gambetta, 1868-1882
}

Volcano speeker Leon Gambetta, 1868-1882

El volcánico Léon Gambetta, 1868-1882

\section{Aude Dontenwille-Gerbaud}

\section{CpenEdition}

\section{Journals}

Édition électronique

URL : https://journals.openedition.org/mots/22331

DOI : $10.4000 /$ mots.2233

ISBN : 978-2-84788-836-2

ISSN : 1960-6001

\section{Éditeur}

ENS Éditions

\section{Édition imprimée}

Date de publication : 10 septembre 2016

Pagination : 33-48

ISBN : 978-2-84788-835-5

ISSN : 0243-6450

Référence électronique

Aude Dontenwille-Gerbaud, « Le volcanique Léon Gambetta, 1868-1882 », Mots. Les langages du politique [En ligne], 111 | 2016, mis en ligne le 10 septembre 2018, consulté le 23 avril 2022. URL : http://journals.openedition.org/mots/22331 ; DOI : https://doi.org/10.4000/mots.22331 


\section{Le volcanique Léon Gambetta, 1868-1882}

Léon Gambetta poursuit sa catilinaire, s'anime, s'exalte, couvre de sa voix sonore la voix de son adversaire, l'anéantit, le submerge - le mot est de lui - et, les cheveux épars, la robe en désordre, ses vêtements ouverts, sa cravate enlevée, son col nu, semblable à un autre 0 'Connel rugissant à la tribune, l'avocat menace, tempête, accuse, écrase et retombe épuisé sur son banc, tandis que des applaudissements éclatent, saluant à la fois la réparation qui commence et le tribun qui s'est révélé. (Gheusi, 1909, p. 266)

Cette description relate l'un des moments, devenu mythique, chez les républicains fondateurs de la Troisième République. Nous sommes sous le Second Empire, Léon Gambetta n'a que 30 ans et il n'est guère connu que dans les cafés du Quartier Latin. Sa plaidoirie au procès du journaliste Delescluze, véritable programme politique, très largement diffusée et commentée, fera de lui le leader des opposants à l'Empire. Dès les premiers témoignages, l'orateur est considéré comme « différent ». Si l'on considère la norme au sens linguistique, quelle conscience normative fait émettre aux locuteurs de l'époque de Léon Gambetta un pareil jugement pouvant aller, dans certains milieux, jusqu'à la nonacceptabilité? Quelle part de ce jugement correspond à la langue elle-même et à d'éventuelles normes prescriptives, quelle importance est accordée aux normes de communication politique? Nous avons à nous interroger sur ce décalage qui fait de Gambetta un leader considéré comme exceptionnel, sans sousestimer le contexte : les normes de communication et les usages en politique sont eux-mêmes en construction. La République n'est pas encore installée, héritière de la Révolution française et d'une Seconde République qui n'a pas duré. Entre 1870 et 1882, une restauration monarchique est encore possible, les partis politiques n'existent pas au sens moderne du terme, la représentation nationale n'est pas encore un univers constitué d'experts (Anceau, 2003).

Comme tous les orateurs du début de la Troisième République cependant, Léon Gambetta a été formé à la rhétorique. Il semble même avoir cherché à se former au mieuxà l'art oratoire, comme en témoignent ses proches, recopiant, apprenant par cœur des discours entiers d'orateurs grecs ou latins. Il va beaucoup écouter, en Sorbonne, à l’École de Droit, à la célèbre conférence Mollé, 
aux conférences positivistes, aux séances du Corps législatif, également aux prêches de Notre-Dame, ou encore à ceux de la chapelle de l'Oratoire.

Pour être en mesure d'analyser le discours politique de Léon Gambetta, une analyse de contenu ne peut suffire. Il nous faut prendre en compte toute la dimension d'oralité des discours du leader et travailler à faire émerger ce rapport aux normes et aux usages dans la matérialité même de la langue. L'historienne Françoise Waquet interroge cette question de l'oralité dans l'historiographie et regrette ce qu'elle nomme une double équation, souvent implicite, selon laquelle l'écrit s'apparente au monde cultivé et savant, alors que l'oral renverrait au monde dit «populaire », à l'exception de l'éloquence de la chaire, de la tribune et du barreau. L'analyse de cette forme d'oralité privilégie alors une analyse en termes d'art, de style, de codification, de norme (histoire littéraire) et non une analyse prenant en compte les pratiques et les usages (Waquet, 2003).

Nous nous inscrivons pour notre part dans une historiographie qui s'intéresse au lien entre écrit et oral, constitutif d'un événement discursif (Guilhaumou, 2006) : ce qu'il convient d'analyser est une posture nouvelle, entre grands rassemblements populaires et comptes rendus de presse. La parole n'est plus seulement celle des tribunes parlementaires. La presse républicaine orchestre ces moments de « rencontre » entre orateurs et larges publics. La presse d'opposition s'en empare pour les dénigrer. La presse écrite s'articule avec cette oralité. Dans cet univers de réflexivité du discours, les publics quels qu'ils soient font partie des sujets d'énonciation. Pour celui qui prend connaissance de ces rassemblements par la presse, les réactions des publics notées par les sténographes et qui figurent dans les retranscriptions sont révélatrices des capacités interprétatives propres à ces publics réalisant un possible de l'horizon d'attente républicain : se montrer sage, homme régénéré donc libre, digne du suffrage universel, tout en héritant du bouillonnement des actes fondateurs de la Révolution. Se montrer Un, quelles que soient par ailleurs les tendances et nuances sur l'échiquier politique qui se met en place. Les sources de l'historien sont fort diverses et constituent un large corpus :

- les discours de Léon Gambetta, dont nous avons une retranscription en 11 volumes par Joseph Reinach, discours parlementaires et discours devant des assemblées extraparlementaires. Les réactions des publics notées par les sténographes font partie du corpus. Ce ne sont que des réécritures, mais diffusées par la presse, lues et commentées, elles participent de l'événement discursif; - des correspondances privées, celles de Léon Gambetta, Juliette Adam, Jules Ferry, Jules Hetzel...;

- des ouvrages de souvenirs évoquant le tribun, que ce soit de la part de proches, de partisans ou d'opposants : Juliette Adam, Léon Cladel, Alphonse Daudet, Ludovic Halévy, Pierre-Barthélémy Gheusi, Camille Meaux, Joseph Reinach, Albert Tournier; 
- la presse, pour laquelle nous avons opéré une sélection en focalisant notre attention sur les moments de discours ayant particulièrement suscité des polémiques. La République française, le journal de Gambetta, et deux quotidiens très hostiles, l'un national, Le Figaro, l'autre de province, L'Écho de l'Ouest. Il serait intéressant de constituer également un corpus d'images, tant celles représentant Léon Gambetta sont nombreuses. Il s'agit toutefois d'un univers à part, celui des caricatures, que nous n'avons pas ici la possibilité de prendre en compte.

Dans un premier temps, nous verrons comment ce qui se dit des pratiques d'orateur de Léon Gambetta donne à voir en creux des usages en vigueur et des normes en cours de construction durant cette période charnière de fondation de la Troisième République.

Dans un deuxième temps, nous montrerons comment le décalage entre ces usages et normes attendus et les pratiques d'orateur de Léon Gambetta s'inscrit dans cet événement discursif fondateur.

\title{
Le jugement d'acceptabilité des contemporains
}

\section{Le langage de Léon Gambetta}

\begin{abstract}
Souvent, en applaudissant cet âpre méridional, qui nous gueulait les harangues volcaniques de l'aîné des Riquetti à l'Assemblée Nationale, et surtout celle de Danton à la Convention avec un assaisonnement inouï de foutre, de bougre et de nom de Dieu, nous sentîmes passer en nos reins le grand frisson des fièvres civiques d'un autre âge, et nous tous, jeunes gens, écœurés par la platitude générale, nous nous dîmes que l'Hercule de la République et le Tombeur de l'Empire avait enfin surgi... (Gheusi, 1909, p. 123-124).
\end{abstract}

Ce témoignage de Léon Cladel (futur nouvelliste) relaté par l'historienromancier Pierre-Barthélémy Gheusi concerne les moments de rencontre entre étudiants au Quartier latin. La vulgarité affichée du lexique restera une image attachée à Léon Gambetta jusqu'à la fin de sa vie. En creux, ces témoignages pointent une forme de malaise : un orateur politique se doit de tenir son rang par un langage approprié. Alphonse Daudet - dont le langage n'est pas moins truculent -, ne supporte pas Léon Gambetta :

Ce Gambetta qu'ils viennent d'élire, je l'ai connu à son arrivée à Paris, à l'hôtel du Sénat. Ils étaient là une bande - à table d'hôte - de méridionaux du vilain midi, trop noir, trop luisant, et grossier, et criard. Ils avaient tous des têtes de cheval. Castagnary me disait quelquefois : «Allons dîner à l'écurie! ». Et nous descendions à la table d'hôte. C'est Gambetta qui menait le chœur. Vous ne pouvez rien vous figurer de plus bavard, de plus hurlant que ça et sans esprit... Il parlait... il parlait... et les gestes... Avec ça, borgne... - Je me suis bien trompé sur lui. Il y avait sous ce bavardage, mais bien en dessous, de l'éloquence... À force de bavarder, de jeter sa 
gourme, il a fini par parler - et très bien dit-on. Je l'ai connu à l'état de pot-au-feu pas écumé. ça bouillait sale. (Daudet, 1897, III, p.142-143)

Il ne semble pas, toutefois, que ce refus des bons usages soit manifeste chez Léon Gambetta dans les instances parlementaires ou dans les conférences devant des publics populaires. On ne le retrouve pas non plus dans sa correspondance, y compris la plus intime. Il est évidemment possible d'évoquer des retranscriptions qui auraient gommé ces prétendues défaillances, ainsi qu'un tri dans les correspondances. En tant que telles, elles seraient signifiantes : les proches, d'une part, la presse en général - même la presse d'opposition -, ne pourraient pas se permettre de retranscrire un discours politique tenu dans un langage considéré comme vulgaire. On ne trouve trace de ce manquement aux usages qu'une seule fois lors d'un meeting à Charonne où l'orateur, empêché de parler, invective son public... Cet épisode, devenu célèbre, fera le tour des rédactions et sera largement commenté. Le 16 août 1881 en effet, des sifflets se font entendre dans une partie de l'assemblée dès l'entrée de l'orateur et Léon Gambetta, excédé, finira par s'en aller. 8000 à 10000 personnes se trouvent alors rassemblées dans un vaste enclos, en partie seulement recouvert d'un toit. Selon La République française (17 août 1881, p. 1), un petit groupe de «meneurs» se serait mêlé à la foule en profitant de l'obscurité pour empêcher Gambetta de parler. Dès la première interruption, l'orateur en appelle au peuple de Paris et à sa dignité. Des centaines de personnes venues l'écouter l'applaudissent et l'acclament. Léon Gambetta semble donc soutenu par une bonne partie de son public : «Citoyens, celui qui siffle est un lâche [...] Silence aux braillards! Silence aux gueulards! Silence à ceux qui n'ont ni pudeur, ni conscience! » (Gambetta, 1881, IX, p. 489) Manquement aux bons usages de la parole publique? Cette vulgarité peut s'analyser comme stratégie d'énonciation. La force illocutoire du discours dit le mépris que l'on doit avoir pour ceux qui se montrent indignes de la République. Les milliers d'autres personnes présentes sont également accusées, mais elles ne sont pas indignes. Les termes employés par Léon Gambetta à leur égard sont durs, mais parfaitement polis : "Comment, vous seriez impuissants à rétablir l'ordre et à assurer la liberté de la tribune? » (Ibid.). Ce qui semble en jeu dans cette interaction, ce que l'orateur cherche à faire toucher du doigt à son public, c'est la fragilité de la République. Dans le cadre de notre hypothèse, l'ethos discursif est alors celui du formateur. La norme sociale est ici celle du comportement respectueux dû à la République elle-même. Sur un plan plus linguistique, tout se passe comme si le parler vulgaire de Léon Gambetta se faisait l'écho de celui des opposants dans le cadre d'une norme descriptive qui donnerait à voir sans détour la violence du moment. En termes de norme prescriptive, l'usage de ce parler vulgaire, acte perlocutoire, aurait en charge la provocation, destinée à faire réagir le public et, au-delà, toute la presse qui ne manquera pas de s'emparer de l'événement. 


\section{Quel langage dans l'arène parlementaire?}

Analysant la rhétorique parlementaire, Jean-Marc Guislin montre comment, dans le souci de discréditer leurs adversaires, les parlementaires n’hésitent pas à reprendre les arguments ou le style opposés pour mieux les démonter (Guislin, 2005). Cette question du «style» n'est pas simplement d'ordre rhétorique et interroge également l'usage au sens linguistique. Ainsi de cet extrait d'une séance à l'Assemblée du 2 juillet 1873 à propos du mot «infirmité » :

Léon Gambetta. - Eh bien je dis que c'est au nom de cette triple considération, au nom de l'infirmité de votre origine... (Violents murmures à droite et cris : À l'ordre! Àl'ordre!)

M. le baron de Vinols. - C'est une insulte à l'Assemblée ! À l'ordre!

M. le Président. - J'invite M. Gambetta à vouloir bien expliquer l'expression dont il vient de se servir...

M. Gambetta. - C'est très simple...

M. Le Président. - ... En parlant de l'origine de l'Assemblée actuelle. L'Assemblée actuelle a assurément l'origine la plus légitime (TB! TB!)

M. Gambetta. - Je crois que je vais répondre...

Un membre à droite. - Rétractez-vous !

D'autres membres. - Laissez parler!

M. Gambetta. - ... de manière à satisfaire même l'impatient qui me dit : « rétractezvous », aux demandes d'explication dont $M$. le Président s'est fait l'interprète.

J'ai dit l'infirmité de l'origine de l'Assemblée - l'expression est peut-être un peu incorrecte au point de vue de la langue... (Rumeurs à droite).

J'entendais par-là que l'Assemblée du 8 février 1871 - et je n'ai pas besoin de retracer ces circonstances, vous les connaissez comme moi - avait été élue dans des conditions de brièveté, de non-communications, de non-vérifications, qui faisaient qu'elle ne ressemblait pas, comme netteté de mandat... (Protestations à droite...)

M. Courbet-Poulard. - Trouvez donc ailleurs une pareille plénitude de mandat!

M. Gambetta. - Cela est certain, Messieurs, et la preuve en est que ce débat ne se clôt jamais, qu'il se continue non seulement entre vous et nous, mais entre vous et le pays (Réclamation à droite et au centre). Il est bien certain que le mandat que vous avez reçu n'était pas complet (nouvelles réclamations). Voilà ce que signifiait le mot que j'ai prononcé. (Gambetta, 1881, IV, p. 34)

Gambetta dit ne pas avoir trouvé le mot juste. Il est évidemment possible de l'analyser comme un dérapage, simple manquement aux usages respectueux de l'arène parlementaire. Ce qui est plus intéressant, c'est qu'il le reconnaît sans difficulté : «l'expression est peut-être un peu incorrecte au point de vue de la langue... ». Notons que pour Léon Gambetta, la langue n'est qu'un point de vue. Le «peut-être » opère un traitement détensif, d'atténuation, forme d'euphémisme pour minimiser la responsabilité du locuteur dans le choix du lexique. Pour autant, s'agit-il d'un ethos de conciliation? Dans le déploiement de l'interaction entre ces parlementaires, ce n'est plus Léon Gambetta mais le débat 
qui continue, ce n'est même plus un débat entre parlementaires, mais entre une partie de ces parlementaires et le pays lui-même, en raison des circonstances historiques. En quelque sorte, on assiste à l'effacement énonciatif de l'orateur en fin d'argumentaire. La question de l'usage n'en est plus une puisque le locuteur n'est plus directement l'énonciateur. Les manifestations de la salle notées par les sténographes ne peuvent donc s'adresser directement à l'orateur.

À l'inverse, c'est parfois Léon Gambetta lui-même qui invoque un parler inadéquat chez l'adversaire : ainsi de cette séance houleuse à l'Assemblée le 30 août 1871. Léon Gambetta défend l'idée d'une Constituante, ce que refusent les non-républicains, qui ne cessent de l'interrompre. L'orateur reste calme, tout au moins d'après la transcription, et se permet même de rappeler à l'ordre des bons usages un représentant bonapartiste :

M. Gambetta. - Un de mes nombreux interrupteurs, M. Pagès-Duport, me passe le bulletin des Lois de la République française, n²2, et me dit d'un air triomphant: «Défendez-vous!». Qu'il m'écoute! (Oh, oh!)

Permettez! Quand je suis attaqué, j'ai le droit de demander qu'on écoute la défense! (Oui! Parlez!)

M. Pagès-Duport ajoute même, dans un langage tout à fait commercial qui lui est familier: (Rumeurs) «Ne laissez pas protester votre signature!»

Je n'en ai pas l'habitude et je vais le lui prouver, à lui qui doit connaître la valeur de ces mots. (Gambetta, 1881, II, p. 84)

Ce mépris affiché par Léon Gambetta pour le langage « commercial » place cette fois l'ethos du tribun du côté des lettrés, de l'avocat garant du langage digne des questions constitutionnelles, lui par ailleurs fils de commerçant. Michel Offerlé montre comment, dans le processus de légitimation, de multiples opérations tendent à accréditer la prétention à l'exercice du pouvoir politique. Il s'agit de mettre en évidence des qualités pour justifier cette prétention, puis transformer cette description (qui ne renvoie qu'à des qualités sociales) en une norme d'accès à la parole politique. De ce point de vue, la bourgeoisie écarte d'avance les candidatures ouvrières. Les vrais autodidactes d'origine populaire sont rares au Parlement (Offerlé, 1984). Même si Léon Gambetta n'est pas un autodidacte, il reste néanmoins le fils du commerçant de Cahors et pas seulement pour ses opposants. Les républicains issus de la moyenne et grande bourgeoisie se méfient également de ce «méridional » (Antonmatei, 1999; Barral, 2008; Mayeur, 2008). Il est évident que des traits non linguistiques accentuent la perception d'écarts proprement discursifs.

\section{Des traits non linguistiques}

Juliette Adam (grande bourgeoisie républicaine) demande l'avis de son ami, l'éditeur Pierre-Jules Hetzel, avant d'inviter Léon Gambetta dans son salon mondain parisien : 
- Est-il invitable, ce pourfendeur de 1848 ? demandai-je.

- Non, impossible. Quand Alphone Daudet m'en parle, il faut l'entendre me dépeindre le clan méridional de Gambetta, clan du «bas midi», composé de gascons criards, de Provençaux péroreurs, bruyants. Lui, une sorte de commisvoyageur en marchandise politique estourbissante, se gobant, provincial jusqu'aux moelles, provincial d'épicerie, borgne avec cela, et habillé et chemisé et cravaté et pantalonné, en dégringolade. (Adam, 1905, p. 305)

Et sa première apparition dans ce salon bourgeois est restée dans les annales : un personnage arrivé en chemise et non en habit... Mélange, sans doute, d'ignorance et de volonté maligne de choquer. N'oublions pas le contexte des mœurs de l'époque. Les écrits de Jules Vallès, par exemple, témoignent de cette importance des codes comportementaux qui interdisent dans son milieu bourgeois le rire, le bruit, toute manifestation de sentiment. Ne pas faire "peuple», respecter la morale bourgeoise dans la famille de Jules Vallès, c'est se montrer mutique et immobile (Périer, 2012, p. 341-342). Faut-il y voir une incapacité, à tout le moins une difficulté du tribun à se mouler dans les usages sociaux voulus par ce milieu bourgeois? Nous proposerons ici une autre hypothèse d'analyse : celle d'une attitude délibérément adoptée et travaillée. Sur le plan discursif, cet ethos et son déploiement dans la matérialité de la langue participerait de l'événement discursif fondateur de la Troisième République.

\section{L'événement discursif fondateur : nouvelles normes républicaines}

\section{Former l'Homme nouveau}

Paula Cossart analyse, à propos des meetings politiques de ces années de fondation républicaine, la contradiction entre un projet de formation d'un citoyen éclairé, capable de débattre, de réfléchir par lui-même, plein de «retenue démocratique » et les effets d'une rhétorique des passions, qui semblerait vouloir dire qu'un orateur habile et convaincant impose ses idées (Cossart, 2010, p.191). Le projet affirmé des Pères fondateurs de la République n'est pas de refuser les élans collectifs, mais d'éduquer le public à n'ovationner que le principe, la République elle-même, en aucun cas l'orateur. Yves Deloye interroge le paradoxe d'un leader charismatique qui incarnerait de manière idéal-typique la figure du mérite républicain : retenue démocratique faite de sagesse, sobriété, maîtrise des émotions et des passions alors même que le charisme du tribun le fait applaudir, parfois à tout rompre, par ses publics. Yves Deloye note que c'est chez Léon Gambetta que l'on observe la plus forte réticence, voire parfois la plus forte hostilité, à l'égard de certaines formes d'expression publiques du mérite et de l'honneur, dès lors qu'elle concerne sa propre personne. Gambetta 
établirait une véritable « grammaire de la déférence », appelant son auditoire à la modération et à la maîtrise des formes de reconnaissance publique de l'estime et du mérite, dans une homologie très forte entre la forme des réunions politiques qu'il préside et le fond des valeurs que le parti républicain et, par extension, la république incarnent. (Deloye, 2000). Nous l'analysons quant à nous comme événement discursif, acte fondateur de la Troisième République. Quelle que soit l'idée républicaine, elle ne vaut que par le lien, l'interaction, la conformation entre les orateurs et leurs publics. Rien n'oblige Léon Gambetta, leader de stature nationale puis président de la Chambre, à se rendre partout en France, tenir des meetings devant des publics déjà acquis à la forme républicaine. La méthode politique est nouvelle et, au-delà de l'usage qu'en fait l'orateur, ce qu'il donne à voir est une nouvelle norme qu'il propose aux républicains de sa génération : celle de la nécessaire épreuve du discours politique devant des assemblées populaires. Les archives et les correspondances témoignent de la difficulté de Léon Gambetta à préparer un discours et de l'importance des publics dans la clarification de ses idées, ce qu'une analyse des discours en termes d'interaction corrobore complètement. À l'évidence, le «bouillonnant» Gambetta qui appelle tout un chacun à s'assagir prêche tout autant pour lui-même que pour ses publics. Eugène Spuller, évoquant son ami, insiste sur l'importance de cette interaction fondatrice :

C'est en vue de cette grande et nécessaire transformation des masses profondes du suffrage universel qu'ont été entrepris tous ces voyages, qu'ont été prononcés tous ces discours d'un si grand effet sur l'opinion [...]. Aussi bien, par une réaction bien naturelle de ces divers auditoires si différents et pourtant si semblables, sur l'orateur qui les entretenait de leurs affaires, il s'est trouvé que la France a enseigné au tribun populaire cette politique de prudence et de mesure, de circonspection et de patience, cette nécessité si impérieuse du bon sens, de la réserve et de la sagacité dans la bonne conduite des choses humaines. Messieurs, laissez-moi vous le dire, la nouvelle méthode qui préside à la direction des affaires du parti républicain, c'est la France qui l'a enseignée, c'est la France qui la veut et qui l'a imposée. (Sensation profonde). (Spuller, 1879, p. 17).

Proscrire l'usage des ovations personnalisées, ce n'est pas seulement dire mais expérimenter ce que doit être l'Homme nouveau, le citoyen républicain. Le peuple de France a trop souffert de la personnalisation du pouvoir. L'épisode bonapartiste est là pour le rappeler. Léon Gambetta le répètera inlassablement, souvent en vain; ainsi de cet exemple à Bonneville, le 30 septembre 1872 :

Je me demande comment il peut se faire qu'après tant et de si éclatantes impostures, il reste encore dans l'âme du peuple assez de générosité, de confiance et d'abandon, pour qu'il continue à prendre un homme et à l'entourer, à l'acclamer, au risque d'en faire une idole dangereuse. Aussi, Messieurs, toutes les fois que j'en trouve l'occasion, et sans rechercher si ma modestie ou l'envie des autres y trouve son compte, ce que je tiens à répéter constamment, c'est que nous devons nous 
déshabituer tous, les uns et les autres, de trop compter sur un homme, de trop le regarder comme un symbole et un drapeau; nous ne devons le considérer que pour ce qu'il vaut, que pour les efforts qu'il fait, mais ne jamais lui donner un rang ou une place qui est toujours disproportionnée à la valeur d'un individu. (Bravo! Bravo!). (Gambetta, 1881, III, p. 140)

Ce discours de Léon Gambetta répond très directement à la presse réactionnaire :

Cet homme au front d'airain, mais sans idée, sans nulle valeur, tient vraiment trop de place. Ce n'est pas naturel, et même l'immense bêtise de ce lumineux $19^{\text {e }}$ siècle n'explique pas suffisamment une pareille exorbitance [...]. Gambetta est le plus vulgaire, et tous se mettent à sa suite, et il prend lui, la tête du mouvement révolutionnaire sans discussion et sans compétition. Il y a là du surnaturel; c'est l'homme du destin, marqué pour quelque mission terrible. (L'Écho de l'Ouest, 15 mars 1872)

\section{S'adapter aux usages de publics divers}

Les publics extraparlementaires de Léon Gambetta ne se comportent pas de façon identique. Un traitement général du corpus des discours extraparlementaires devant ces publics a permis de déterminer un indice de réactivité. Le calcul en est simple : compter toutes les marques de réaction des publics notées par les sténographes et diviser ce nombre par celui des pages du discours transcrit. Ce ne sont que des tendances et il ne sera jamais possible d'affirmer la réalité de ces réactions qui sont d'abord l'appréciation des sténographes présents. Comme nous l'avons montré dans une étude portant sur «les publics populaires» (Dontenwille-Gerbaud, 2015), ce qui distingue Paris de la province, ce sont les comités électoraux et les élus, les élites républicaines par conséquent. Ils réagissent davantage que les électeurs eux-mêmes. En province, à l'inverse, la masse des électeurs, public rural ou public mixte, semble plus bruyante que celle des élus. La concurrence entre candidats politiques explique sans doute le côté agité, prompt aux applaudissements ou aux ovations du public des comités parisiens. Lorsqu'en revanche la réunion est plus vaste, concentre le maximum possible d'électeurs, le public semble beaucoup plus calme, même à Belleville. Le public rural, quant à lui, qui peut venir de très loin (parfois une centaine de kilomètres à pied) semble globalement plus silencieux que le public urbain. Il réagit plus rapidement, a tendance à répondre plus directement à l'orateur: les « Oui ! Oui ! », les « C'est vrai ! », les «C'est cela!» sont nombreux. Ce public, toutefois, ne manifeste aucune réaction durant de longs moments de discours. Enfin, le public le moins bruyant est composé des négociants, des commerçants et des industriels. À la fin des discours, cependant, ce sont des salves répétées d'applaudissements, des acclamations. Les assistants se pressent autour de l'orateur pour le saluer et le féliciter. Léon Gambetta ne cesse de s’adapter. Un de ses amis en témoigne: 
Tandis qu'il se rendait à la Ferté-sous-Jouarre, un ami lui ayant demandé s'il était prêt, il répondit : "Je ne sais pas ce que je dirai ; il faut d'abord que je voie la réunion : la forme de mon discours variera selon que l'auditoire sera paysan, citadin ou mixte». (Tournier, 1893, p. 328)

Cette méthode politique nouvelle sera raillée, Léon Gambetta traité de "commis-voyageur de la République », ce qu'il revendiquera alors avec humour comme méthode politique. Ainsi, le 9 octobre 1878, les voyageurs de commerce, de passage à Grenoble, envoient une délégation à Léon Gambetta :

Vous avez été pour la République, on ne le sait pas assez, les meilleurs messagers de la bonne nouvelle. Vous l'avez fait pénétrer dans les endroits les plus écartés, dans ceux où le journal n'arrivait pas, où la parole des hommes politiques ne pénétrait qu'affaiblie [...] Vous êtes les plus précieux collaborateurs de la démocratie, et je ne peux pas oublier que, moi aussi, on m'a appelé le commis-voyageur de la République (Salves d'applaudissements et rires). Non, Messieurs, je n'ai pas oublié cette parole, et j'ai considéré que ceux qui prétendaient ainsi m'adresser une injure étaient des impuissants et des sots.

Je vous ai imités. J'ai parcouru la France en portant le germe qui se féconde, qui croît tous les jours, mais pour le développement duquel il faut des collaborateurs. Enfin, vous avez semé, Messieurs. La moisson lève; nous récolterons tous ensemble. (Assentiments unanimes et applaudissements.) (Gambetta, 1881, VIII, p. 255-256)

«Le germe qui se féconde », «la moisson qui lève»: le champ sémantique évoque la France des campagnes qui est à convaincre politiquement et le leader n'est rien sans les collaborateurs. Ceux qui méprisent ce nouvel usage de la parole politique, cette interaction fondatrice, ne sont que des impuissants et des sots. Les termes sont forts et sans appel. Ce n'est pas la méthode de Léon Gambetta qui est alors méprisée, mais bien une forme nouvelle de lien entre hommes politiques et citoyens ; l'interaction prend une dimension sacralisée, celle des messagers de la bonne nouvelle.

\section{Quels usages de la rhétorique?}

Comparons quelques orateurs républicains : Paul Bert, Jules Ferry, Charles Floquet, Léon Gambetta, Victor Hugo, Eugène Spuller. Prenons comme indicateur les marques de "sensation», «mouvement», "émotion» ou bien celles du «rire», "hilarité», "sourire» notées par le sténographe dans les discours devant des publics extraparlementaires (Dontenwille-Gerbaud, 2006, p. 82-83). La fréquence de réaction des publics lors des discours de Léon Gambetta se montre largement plus importante que celles des publics des autres orateurs républicains. Le tribun cherche à établir un lien avec son public et à le faire réagir. Les Anciens, ceux de 1848 (Louis Blanc, Victor Hugo) utilisent beaucoup le registre de l'émotion et peu celui du rire. Les plus jeunes (Paul Bert, Eugène Spuller, Léon Gambetta) utilisent tout autant l'un et l'autre, sauf 
Jules Ferry, le positiviste qui s’inscrit dans une tradition anti-rhétorique. Ces résultats disent aussi, du point de vue des usages, la bataille entre républicains analysée par Françoise Douay-Soublin (1999). Léon Gambetta semble donc susciter des interactions plus directes avec ses publics et pour autant, rien ne permet de constater qu'il utilise les registres du pathos ou du rire différemment des autres orateurs républicains de sa génération. Léon Gambetta, dès 1869, considère que Jules Ferry n'a pas assez d'action sur le «populo». A contrario, Jules Ferry s’inquiétera plus tard des «intempérances » de Léon Gambetta, qui risquent de nuire à la cause républicaine. André Lavertujon, comme beaucoup de ses contemporains, s’interroge à propos de Jules Ferry :

L'observation est exacte, je dois le reconnaître [...]. Je l'ai constaté au Corps Législatif et pendant le Siège; mieux encore plus tard, pendant le grand ministère Ferry, où les journaux, les grands orateurs de réunion, les caricaturistes avaient réussi à faire de lui un être exceptionnellement exécré [...]. Mais je ne dois parler ici que de l'impression qu'on recevait de sa personne, de son habitude de corps, de son geste, de sa voix; généralement elle n'était pas favorable [...]. C'est sa différence avec Gambetta, dont la sympathie débordante entraînait tout. (Lavertujon, 1905, p. 142)

Léon Gambetta joue-t-il de cette sympathie pour faire accepter toute forme d'évolution, voire de reniement de ses promesses? Un discours devenu célèbre, prononcé à Belleville le 23 avril 1875, est présenté par les historiens comme celui du grand jeu rhétorique. De fait, dans un discours prononcé le 28 février 1873 devant l'Assemblée Nationale, Léon Gambetta avait condamné catégoriquement l'idée d'un Sénat dans la constitution républicaine. Or le 23 avril 1875, devant le public populaire de Belleville, il démontre le bien-fondé d'une seconde Chambre alors même que la tradition républicaine est hostile, par principe, à l’idée d'un Sénat. Chloé Gaboriaux analyse le lien que Gambetta opère, à ce moment précis des luttes politiques, entre le «système électoral » et le «système social», entre la distribution du pouvoir politique et l'organisation du monde social. L'adoption du Sénat, peu après le vote des lois constitutionnelles, était le prix à payer (Gaboriaux, 2015). L'analyse en histoire politique montre comment les arguments, d'abord stratégiques, vont devenir des arguments de principe : Gambetta invite à tirer les leçons des transformations sociales qui affectent le personnel politique des communes et des départements. L'historien en analyse du discours peut préciser comment fonctionne, pas à pas, l'interaction entre l'orateur et le public populaire de Belleville pour faire accepter cette évolution (Dontenwille-Gerbaud, 2013). Ne prenons ici que la chute du discours. Elle illustre en quelque sorte la dimension hors norme de l'orateur :

Vous voyez que, lorsqu'on fait de la politique d'intrigue, on est très souvent pris à ses propres pièges (Rires). On s'est trompé, l'étiquette a tout perdu : on appelait cela un Sénat, et on a cru qu'on aurait un Sénat (Rires). 
Mais énumérez les conditions de l'institution, regardez-y bien près, voyez ces communes éveillées à la vie politique, se groupant, se réunissant, se renseignant, s'informant, délibérant, déléguant leurs hommes; ceux-ci s'assemblant au cheflieu du département, faisant prévaloir leurs volontés, lesquelles seront, le jour de l'élection, ce qu'elles auront été la veille. Après la délibération commune, que va-t-il sortir des urnes? Un Sénat? Non, citoyens, il en sortira le Grand Conseil des Communes françaises (Applaudissements). (Gambetta, 1881, IV, p. 318)

Cette formulation fait partie de celles qui ont obtenu un très fort retentissement à l'époque.

Aujourd'hui, elle est régulièrement citée pour démontrer l' "opportunisme » de Léon Gambetta. Durant près de deux heures, la formulation de Grand Conseil des communes française s'élabore peu à peu, au milieu des rires et des applaudissements, cherchant à établir une définition générique de la commune et du Sénat qui soit recevable en République. Au sein des différents effets lexicaux créés par Gambetta, cette formulation apparaît comme une des rares qui soit positive. Il est possible de l'analyser comme une surassertion par lequel un fragment se présente dans le discours comme détachable. Gambetta, en homme politique parfaitement novateur, utilise ici ce que l'on peut qualifier de «petite phrase » au sens développé par Alice Krieg-Planque (2011, p. 23-41). Cet objet est coproduit par les médias et les politiques. L'orateur sait parfaitement que l'ensemble de la presse s'emparera non pas tant de la rhétorique déployée que de l'énoncé «Grand Conseil des Communes de France». Cette «petite phrase » s'inscrit dans l'événement discursif fondateur, souligné rhétoriquement par l'utilisation du pathos:

Alors ils se diront les uns aux autres le grand secret de la politique démocratique, car cette politique a un secret (Mouvement). Oh! il ne faut pas le cacher, ce secret; il faut, au contraire, l'apprendre, le communiquer, le répandre, le divulguer à tous. Ce secret, le voici. Faire comprendre à celui qui dispose d'un bulletin de vote tout ce qu'il y a dans ce petit carré de papier. (Gambetta, 1881, IV, p. 319)

Il aurait été possible au leader républicain de présenter son argumentation dans un article de son journal La République française. Créer et faire circuler sa «petite phrase» qui fera date s'élabore ici au sein d'une interaction. Elle sera ensuite, bien évidemment, largement reprise, commentée par la presse. Mais le coup de tonnerre politique est légitimité par l'accueil du public, et pas n'importe quel public puisqu'il s'agit du celui de Belleville, le plus révolutionnaire des publics républicains. Il est certes possible d'analyser ce type de discours comme une simple manipulation à l'aide d'une rhétorique bien rodée. Il est encore possible d'évoquer une claque présente au sein du public (les archives, toutefois, ne permettent pas de l'attester et la presse d'opposition elle-même ne l'évoque pas). Il est aussi possible d'analyser un rapport autre au discours politique, un événement discursif instituant une nouvelle norme inhérente à la République elle-même : ce ne sont plus seulement les Parle- 
mentaires qui agissent, mais la légitimité de leurs prises de décision se rapprocherait d'une coproduction du discours entre hommes politiques, presse et publics. La presse opposée à Léon Gambetta ne manque pas d'ironiser sur cette démarche hors norme :

Alors Gambetta se lève, ouvre la bouche et parle pendant 17 colonnes de la République Française. Quelquefois il est lancé, comme avant-hier; il cascade, il en dit trop. Alors Spuller le pousse par le bras et lui dit tout bas : «En voilà assez; nous avons déjà 3 pages du journal; il ne restera plus de place pour les annonces». Alors Gambetta s'arrête et lance la tirade de la fin contre Monsieur Buffet, chante le couplet final à la République, reçoit les applaudissements du public et va ôter son rouge. (Le Figaro, 26 avril 1875)

\section{« La forme emporte et résout le fond"}

Pour conclure, Léon Gambetta modifie l'usage du discours politique, dans une démarche nouvelle. II met en cause, par sa pratique même des meetings, une norme parlementaire en allant chercher légitimité à l'extérieur de l'arène parlementaire. Cet usage nouveau se traduit dans la matérialité même de la langue, ce qu'analyse Joseph Reinach, l'ami de Léon Gambetta à l'origine de la publication des onze volumes de discours :

La langue du XVIII siècle claire et vive comme l'eau de roche avec Voltaire, majestueuse et chargée de métaphores avec Rousseau, d'une nerveuse et prégnante brièveté avec Montesquieu, ce n'est pas la sienne. La sienne est celle du Xvıe, le parler large et plein qui puise les mots dans le riche et robuste lexique du peuple, les pousse en avant, drus et nombreux, sans autre méthode que celle qui est nécessaire à marquer le progrès des idées, étale les images, surabonde de belle humeur et appelle toutes les choses par leur nom. L'expression énergique et pittoresque est souvent un peu grosse; le flux des paroles est parfois tumultueux; les images sont parfois banales. Mais les images qui sont usées chez l'écrivain se rajeunissent à l'éclair rapide qui jaillit de la tribune, la sûreté de la pensée remet dans le désordre des mots une harmonie supérieure, et les mots eux-mêmes, les plus vulgaires et les plus rudes, emplissent la bouche et éveillent les échos. Que cette haute graisse d'éloquence, si l'on peut traduire l'oratoria ubertas des Latins, ne soit pas souvent compatible avec la correction académique ou même grammaticale, ceci n'a rien qui doive surprendre. Même dans les deux dernières années de sa vie, bien que son style se fût beaucoup allégé et que sa phrase, sans rien perdre de son ampleur, se dégageât dans une construction tous les jours plus régulière, Gambetta l'avouait en riant : «Je ne travaille point, disait-il, pour le Courrier de Vaugelas». (Reinach, 1881, XI, p. 303-304)

Joseph Reinach évoque un parler gaulois, un retour à Rabelais qu'il met en relation avec l'innovation politique que représente le leader. La référence à Vaugelas est intéressante en termes de normes et d'usage du discours politique. Zygmunt Marzys fait remarquer que l'expression «bon usage » n’apparaît que 
tardivement, dans une adjonction de Préface qui a pour objectif une attitude moraliste au sens social du «qu'en-dira-t-on » (Marzys, 2010). Elle s'oppose aux théoriciens du $16^{\mathrm{e}}$ siècle pour lesquels la normalisation est à venir en élevant la langue vulgaire au statut de langue classique. Pour Zygmunt Marzys, l'idée même que des normes soient à rechercher dans le présent et non dans un développement serait ensuite reprise par la bourgeoisie française, puis relayée par l'École, notamment républicaine. En ironisant à propos de Vaugelas, en renouant aux dires de Reinach avec un parler qui évoque celui du $16^{e}$ siècle, Léon Gambetta se situe aussi dans cette vision d'avenir. "La forme emporte et résout le fond », ne cessera-t-il de proclamer. Les historiens analysent cette assertion en termes politiques comme «la République dans sa forme constitutionnelle résoudra peu à peu la question sociale », ce qui est certainement très juste mais n’épuise pas la pensée gambettiste. La forme que prend son éloquence est aussi action, force perlocutoire pour faire émerger un autre rapport au politique. Ce qui fait du volcanique Gambetta un homme politique à part.

\section{Sources}

Discours et plaidoyers politiques de Gambetta (1881) rassemblés par Joseph Reinach, Paris, Charpentier (11 volumes).

L'Écho de l'Ouest, 1872.

Le Figaro, 1875.

La République française, 1881.

AdAm Juliette, 1905, Nos sentiments et nos idées avant 1870, Paris, Lemerre.

DAUDET Alphonse, 1897-1899, Notes du Carnet Papillon, partiellement reproduites dans Fuvres complètes, volume III, 2, Paris, Fayard.

GHEUSı Pierre-Barthélémy, 1909, Léon Gambetta par Léon Gambetta. Lettres intimes et souvenirs de famille, Paris, Société d'Éditions littéraires et artistiques.

LaVertujon André, 1905, Gambetta inconnu. Cinq mois de la vie intime de Gambetta, Bordeaux, G. Gounouilhou.

REINACH Joseph, 1881, "Gambetta orateur », Discours et plaidoyers politiques de Gambetta, Paris, Charpentier, vol.XI, p. 299-318.

SPULLER Eugène, 1879, Conférences populaires, Paris, Dreyfous.

TOURNIER Albert, 1893, Gambetta : souvenirs anecdotiques, Paris, Marpon et Flammarion.

\section{Références}

AnCEAU Éric, 2003, "Les écoles du Parlement. Les types de formation des parlementaires », Les Parlementaires de la Troisième République, J.-M. Mayeur, J.-P. Chaline, A. Corin éd., Paris, Publications de la Sorbonne, p.167-195.

Antonmattei Pierre, 1999, Gambetta, héraut de la République, Paris, Michalon. 
BARral Pierre, 2008, Léon Gambetta, Tribun et stratège de la République (1838-1882), Toulouse, Privat.

Cossart Paula, 2010, Le meeting politique. De la délibération à la manifestation (18681939), Rennes, PUR.

- 2003, "L'émotion: un dommage pour l'idée républicaine. Autour de l'éloquence de Léon Gambetta », Romantisme, nº119, p. 47-60.

Deloye Yves, 2000, «Le charisme contrôlé », Communications, n6 69, p. 157-172.

DeLoYe Yves, IHL Olivier, 2000, «Deux figures singulières de l'universel : la République et le sacré», La démocratie en France, vol. I, M. Sadoum éd., Paris, Gallimard, p.138-246.

Dontenwille-Gerbaud Aude, 2006, «Les discours, acte de fondation de la République. L'interaction orateur/publics populaires. Eugène Spuller, Charles Floquet, Louis Blanc à Troyes en 1879 ", Revue d'Histoire du xIxe siècle, nº33, p. 79-100.

- 2013, «Interactions orateurs/publics populaires. Acte de fondation de la Troisième République », L'art de gouverner. Questions éthiques et politiques, A. Louis, A. Revel éd., Berne, Peter Lang, p. 175-192.

- 2015, «Typologie des réactions des publics populaires républicains lors des grands meetings politiques, 1871-1882 ", À la recherche des publics populaires, Nancy, Éditions universitaires de Lorraine.

DouAY-Soublin Françoise, 1999, «La rhétorique en France au XIXe siècle à travers ses pratiques et ses institutions. Restauration, renaissance, remise en cause», Histoire de la rhétorique dans l'Europe moderne, 1450-1950, M. Fumaroli éd., Paris, PUF, chap. 23.

GABORIAux Chloé, 2015, «Fonder la République sur les "nouvelles couches sociales” (Gambetta). Description du monde social et préférences institutionnelles dans la France des années 1870 », Histoire@Politique, n² 25, en ligne (http://www.histoirepolitique.fr).

Guilhaumou Jacques, 2006, Discours et événement. L'histoire langagière des concepts, Besançon, Presses universitaires de Franche-Comté.

GUISLIN Jean-Marc, 2005, "L'éloquence parlementaire aux débuts de la Troisième République», Parlement[s], Revue d'histoire politique, Paris, L'Harmattan, nº3, p. 39-60.

KRIEG-PLANQUe Alice, 2011, "Les "petites phrases" : un objet pour l'analyse des discours politiques et médiatiques», Communication et langages, n ${ }^{\circ} 168$, p. 23-41.

MAYEUR Jean-Marie, 2008, Léon Gambetta. La Patrie et la République, Paris, Fayard.

MARZYS Zygmunt, 2010, “Le bon usage” et “la plus saine partie” dans les Remarques de Vaugelas», Vox Romanica, n69, p. 188-205.

OffERLÉ Michel, 1984, "Illégitimité et légitimation du personnel politique ouvrier», Annales. Économies, Sociétés, Civilisations, n²4, p. 681-716.

PARmén IE Antoine, Bonn IER DE LA ChAPELLE Catherine, 1953, Histoire d'un éditeur et de ses auteurs. P.J. Hetzel (Stahl), Paris, Albin Michel.

PÉRIER Maguelone, 2012, "Vallès et la chanson : le geste, la voix et le cri du peuple», Presse, chanson et culture orale au xıxe siècle. La parole vive au défi de l'ère médiatique, Paris, Nouveau Monde, p. 341-364.

WAQUET Françoise, 2003, Parler comme un livre. L'oralité et le savoir (XVIe-xxe siècle), Paris, Albin Michel. 
